The Impact of Large-Scale Surveys on Pulsating Star Research

ASP Conference Series, Vol. 203, 2000

L. Szabados \& D. W. Kurtz, eds.

\title{
Unusual Secondary Variations in the Mira Star T Cep
}

\author{
Vladislava I. Marsakova, Ivan L. Andronov \\ Department of Astronomy, Odessa State University, \\ T. G. Shevchenko Park, Odessa 270014, Ukraine
}

\begin{abstract}
Cyclic period variations $\left(P \approx 19400^{\mathrm{d}}\right)$ in $\mathrm{T}$ Cep were investigated. As the result of cross-correlation analysis, relations between individual cycle parameters were found. Relations between period-amplitude, hump-amplitude, hump-minimum magnitude, and others have been obtained.
\end{abstract}

In the interval JD $2423000-2450500$, the period variations of $\mathrm{T}$ Cep are appear to be cyclic. Similar cyclic period changes were detected in W Lyr (Marsakova \& Andronov 1997) and some other Mira variables. But in T Cep these variations are most clear, and are followed by significant variations of amplitude and other individual cycle characteristics.

We have used the amateur observations from the AFOEV and VSOLJ databases (Schweitzer 1998; Nogami 1998) obtained during a 75-yr interval to analyze the period changes and the variability of individual cycle characteristics. The time series analysis was similar to that applied to U Her (Marsakova $\&$ Andronov 1998).

To analyze period changes we have used the $\mathrm{O}-\mathrm{C}$ technique. The shape of the variations is almost sinusoidal; approximation by a trigonometrical polynomial fit of first degree with a reduction of the trend shows $P_{\text {secondary }}=$ $19400^{\mathrm{d}} \pm 400^{\mathrm{d}}$ with an amplitude of $166^{\mathrm{d}} \pm 2^{\mathrm{d}}$.

The parameters which vary most significantly (period, amplitude, asymmetry, magnitudes of maximum, minimum and hump) were studied by using cross-correlation analysis. In this case we have not used the original curves of variation of the individual cycle parameters, but their "running parabolae" (Andronov 1997) fits derived with filter half-width $\Delta t=10500^{\mathrm{d}}$ and a step of $200^{\mathrm{d}}$. The value $\Delta t=10500^{\mathrm{d}}$ was chosen from a signal-to-noise criterion for the majority of the parameters. The results are listed in the Table 1.

As can be seen from the Table, the amplitude is better-related to the magnitude of minimum. Asymmetry changes precede changes of the magnitude of minimum by 5 cycles. The hump is also related to the magnitude of minimum (almost without any shift in time) and with amplitude. Moreover, with decreasing amplitude, the hump becomes like a secondary maximum from the little step in high amplitude state.

In the Cepheid variables, there is a gap in the period-amplitude diagram (Efremov 1968) which is caused by the appearance of a second wave (with the appearance of the hump or a secondary maximum at the light curve). In this case, the maximum becomes more flat and faint. But, in our case, the magnitude 
Table 1. Results of the cross-correlation analysis of the light curve parameters of T Cep. $\rho$ is the correlation coefficient, shift is the shift of parameter 2 from parameter 1 , measured in cycles.

\begin{tabular}{llcc}
\hline parameter 1 & parameter 2 & $\rho$ & $\begin{array}{c}\text { shift, } \\
\text { cycles }\end{array}$ \\
\hline magnitude of min. & magnitude of max. & 0.58 & 22 \\
magnitude of max. & amplitude & 0.66 & 27 \\
magnitude of max. & period & 0.68 & 23 \\
magnitude of min. & asymmetry & 0.79 & -5 \\
magnitude of hump & amplitude & 0.85 & 0 \\
magnitude of hump & asymmetry & 0.85 & -4 \\
magnitude of min. & magnitude of hump & 0.87 & -1 \\
magnitude of min. & period & 0.89 & -5 \\
amplitude & period & 0.93 & -3 \\
magnitude of min. & amplitude & 0.98 & 0 \\
\hline
\end{tabular}

of the hump is better-related to a minimum than a maximum, and this argument breaks the analogy with Cepheids.

The minimum appears to be the main stable state of Miras (Mennessier 1980), so the appearance of the hump is related to processes which change brightness in the stable state of the star. The 3-cycles shift in the period-amplitude dependence is also interesting.

It is possible to discuss two hypotheses to explain behavior of $\mathrm{T}$ Cep:

1) The star is a component of double system (but this hypothesis does not explain the variation of the shape of hump);

2) there is interference of pulsations with two periods. Theoretical modeling of the light curves is needed to check these hypotheses.

Acknowledgments. Authors are thankful to amateur astronomers from the AFOEV and VSOLJ for their observations and to the organizing committee and IAU for the travel grants.

\section{References}

Andronov, I. L. 1997, A\&AS, 125, 207

Efremov, Ju. N. 1968, Perem. Zvezdy, 16, No. 4, 365

Marsakova, V. I. \& Andronov, I. L. 1997, Kinem. Phys. Cel. Bodies, 13, No.6, 49

Marsakova, V. I. \& Andronov, I. L. 1998, Ap\&SS, 257, 49

Mennessier, M. O. 1985, A\&A, 144, 463

Nogami, D. 1998, http://ww. kusastro.kyoto-u.ac.jp

Schweitzer, E. 1998, ftp://cdsarc.u-strasbg.fr/pub/afoev/ 